\title{
A Novel Approach to a Piezoelectric Sensing Element
}

\author{
M. Martinez ${ }^{1}$ and A. Artemev ${ }^{2}$ \\ ${ }^{1}$ Institute for Aerospace Research, National Research Council of Canada (NRC), 1200 Montreal Road, Building M-3, \\ Ottawa, ON, Canada K1A OR6 \\ ${ }^{2}$ Department of Mechanical and Aerospace Engineering, Carleton University, Ottawa, ON, Canada K1S 5B6
}

Correspondence should be addressed to M. Martinez, marcias.martinez@nrc-cnrc.gc.ca

Received 16 September 2010; Accepted 3 December 2010

Academic Editor: K. Kalantar-Zadeh

Copyright (C) 2010 M. Martinez and A. Artemev. This is an open access article distributed under the Creative Commons Attribution License, which permits unrestricted use, distribution, and reproduction in any medium, provided the original work is properly cited.

\begin{abstract}
Piezoelectric materials have commonly been used in pressure and stress sensors; however, many designs consist of thin plate structures that produce small voltage signals when they are compressed or extended under a pressure field. This study used finite element methods to design a novel piezoelectric pressure sensor with a C-shaped piezoelectric element and determine if the voltage signal obtained during hydrostatic pressure application was enhanced compared to a standard thin plate piezoelectric element. The results of this study demonstrated how small deformations of this C-shaped sensor produced a large electrical signal output. It was also shown that the location of the electrodes for this sensor needs to be carefully chosen and that the electric potential distribution varies depending on the poling of the piezoelectric element. This study indicated that the utilization of piezoelectric materials of different shapes and geometries embedded in a polymer matrix for sensing applications has several advantages over thin plate solid piezoelectric structures.
\end{abstract}

\section{Introduction}

Pressure gauges of different design types (e.g., U-shaped tube gauges, piston gauges, aneroid gauges, Bourdon tube, or diaphragm gauge $[1,2]$, optical fiber sensors [3, 4], different electronic sensors) are currently used in different applications. Electronic sensors are convenient because they allow for easy, direct integration into electronic control schemes which can be easily miniaturized and have a short response time when used under dynamic conditions. Electronic pressure sensors can be designed by using different electromechanical or magnetomechanical effects. Electronic sensor types include piezoelectric [5], piezoresistive [6], capacitive, magnetic (inductive), potentiometric, resonant, and surface acoustic wave sensors. In the fields of robotics and orthotics the McKibben actuators have been utilized to mimic the behavior of biological muscles. Son and Goulbourne, 2009 [7] showed how the use of the two electrical parameters capacitance or resistance could be used to measure the large strains/pressure of the actuating device. MEMS based on piezoresistive pressure sensors have also been considered for pressure measurement applications; however they posses low sensitivity and suffer thermal drift $[6,8]$. Piezoelectric pressure sensors are commonly used in sensor designs due to their high reliability and robustness, large range of measurable pressure, and low sensitivity to the electro-magnetic field. In traditional piezoelectric pressure sensor designs, the piezoelectric element is manufactured as a relatively simple shape such as a disc or plate and is subjected to a simple compressive or tensile load applied by the force collector, such as a diaphragm [5, 9-12]. In more sophisticated designs, the piezoelectric film or thin plate is attached to the substrate and subjected to flexure deformations $[13,14]$ which produce a combination of bending and stretching deformation in the piezoelectric component and significantly enhance the piezoelectric response [14]. Recently, it has been demonstrated that the bending of piezoelectric nanowires or nanobelts can produce a very strong electric potential output [15-17] which can be used for energy harvesting. One problem which can be faced with practical implementation of piezoelectric materials in sensor or structural applications is low toughness and high probability of brittle fracture. In that respect the use of piezoelectric materials in form of thin fibers or thin plates 
embedded in polymer matrix is similar to the use of carbon fibers that are brittle as individual component but very resilient when these are embedded as part of a structure in an epoxy matrix. Thus, the widespread use of piezoelectric materials either as structural components or sensors can be resolved by using piezoelectric ceramics in composite materials [18-20].

In the present paper we analyze the possibility of a pressure sensor design in which the shape of the stiff piezoelectric component embedded into the compliant polymer facilitates the bending mode of the piezoelectric sensor deformation when hydrostatic pressure is applied to the composite sensor structure. We consider a relatively simple C-shaped piezoelectric element embedded into a cubic shaped polymer matrix (Figure 1). It is important to note that this study focuses on the piezoelectric element of the pressure sensor which can then be incorporated into the different sensor designs employed to augment the voltage output of the sensing elements as found in the literature [11, 21]. Finite element analyses (FEAs) are used to evaluate the response of this novel sensor design to applied pressure and compare it to the response of a conventional plate-shaped piezoelectric element (Figure 2) subjected to the same pressure conditions.

\section{FEA of a Piezoelectric Sensor}

The FEA of an electromechanical system is based on the mechanical and electrical equilibrium equations:

$$
\begin{gathered}
\nabla \sigma+b=0, \\
\nabla D=0
\end{gathered}
$$

where $\nabla$ represents the divergence operator, $\sigma$ is the stress, $b$ represents the body forces, and $D$ is the electrical displacement. Material properties are presented in the model by the constitutive equations in the stress-charge form:

$$
\begin{aligned}
\sigma & =\bar{C} \varepsilon-e E, \\
D & =e^{T} \varepsilon+\kappa E,
\end{aligned}
$$

where $\bar{C}$ is the elastic material matrix, $e$ and $e^{T}$ are the piezoelectric material matrix and its transpose, respectively, $\kappa$ is the permittivity matrix, $\mathcal{E}$ is the strain, and $E$ is the electrical field. Material parameters used in (2) can be presented in a matrix form as

$$
C=\left[\begin{array}{cc}
- & e \\
e^{T} & \kappa
\end{array}\right] .
$$

The finite element formulation for the linear piezoelectric analysis, derived by using the principle of virtual work, is shown in:

$$
\int\left(B^{T} C B d V\right) X=\int N^{T} p d S+\int N^{T} b d V
$$

where $B$ is the matrix that maps displacement to strain, $N$ is the interpolation matrix, $X$ is the displacement and electric potential vector, $b$ represents the body forces acting on the system, and $p$ is the surface traction vector.

By using (4), an FEA solver has been developed to calculate the displacement and electric potential $(X)$, which can then be used to compute the strain and electric field [20]. Once the strain and electric field are obtained, the stress and electric displacement can be calculated by using (2). The solver uses the Intel Math Kernel Library Sparse Solver for both direct and indirect solutions. All simulations have been performed on a Windows Workstation with an Intel Core 2 Extreme Processor $(2 \mathrm{GHz})$ and 8 Giga bytes of RAM. All the models have been analyzed using the direct solver. The inhouse FEA code makes use of both the 8- and 20-node brick linear elastic and piezoelectric hexahedral elements.

\section{FEA Model Parameters}

The geometry of the composite sensor with a C-shaped piezoelectric component is shown in Figure 1. The C-shaped piezoelectric element had a thickness of $50 \mu \mathrm{m}$ and was embedded into a polymer cube of $1.1 \times 1.1 \times 1.1 \mathrm{~mm}$. The mesh of the piezoelectric element and the polymer matrix was constructed using 20-node brick piezoelectric elements. However, the material properties of the polymer matrix corresponded to those of the polymer with null piezoelectric constants. The boundary conditions were set to provide $100,000 \mathrm{~Pa}$ of pressure uniformly applied to the surfaces of the polymer (hydrostatic pressure). Boundary conditions were applied to prevent rigid body motion and rotation. A single node at the corner of the polymer cube was assigned a zero volt electric potential. The material properties of the piezoelectric component of the sensor were modeled with PZT-5A elements using the stress-charge form of the constitutive equations with the following values: $\stackrel{-}{C}_{11}=\bar{C}_{22}=$ $1.203 \cdot 10^{11} \mathrm{~N} / \mathrm{m}^{2}, \bar{C}_{12}=\bar{C}_{21}=7.518 \cdot 10^{10} \mathrm{~N} / \mathrm{m}^{2}, \bar{C}_{13}=\bar{C}_{23}$ $=\bar{C}_{31}=\bar{C}_{32}=7.509 \cdot 10^{10} \mathrm{~N} / \mathrm{m}^{2}, \bar{C}_{33}=1.109 \cdot 10^{11} \mathrm{~N} / \mathrm{m}^{2}$, $\bar{C}_{44}=\bar{C}_{55}=2.105 \cdot 10^{10} \mathrm{~N} / \mathrm{m}^{2}$, and $\bar{C}_{66}=2.257 \cdot 10^{10} \mathrm{~N} / \mathrm{m}^{2}$. The piezoelectric properties were modeled as $e_{31}=e_{32}=$ $-5.351 \mathrm{C} / \mathrm{m}^{2}, e_{33}=15.783 \mathrm{C} / \mathrm{m}^{2}$, and $e_{24}=e_{15}=12.295 \mathrm{C} / \mathrm{m}^{2}$. The permittivity was set to $\kappa_{11}=\kappa_{22}=8.137 \cdot 10^{-9} \mathrm{~F} / \mathrm{m}$ and $\kappa_{33}=7.319 \cdot 10^{-9} \mathrm{~F} / \mathrm{m}$. In the first model the polarization orientation was set to follow the C-shaped contour of the sensor element, changing discretely between the sections of the sensor element shown with different colors in Figure 1. A second model of the C-shaped embedded sensor with the polarization oriented through the thickness of the sensor was also studied. The properties of the polymer matrix were modeled using the stress-charge form of the constitutive equations with the following values: $\bar{C}_{11}=\bar{C}_{22}=4.83$. $10^{9} \mathrm{~N} / \mathrm{m}^{2}, \bar{C}_{12}=\bar{C}_{21}=2.96 \cdot 10^{9} \mathrm{~N} / \mathrm{m}^{2}, \bar{C}_{13}=\bar{C}_{23}=\bar{C}_{31}=$ $\bar{C}_{32}=2.96 \cdot 10^{9} \mathrm{~N} / \mathrm{m}^{2}, \bar{C}_{33}=4.83 \cdot 10^{9} \mathrm{~N} / \mathrm{m}^{2}$, and $\bar{C}_{44}=\bar{C}_{55}$ $=\bar{C}_{66}=9.348 \cdot 10^{8} \mathrm{~N} / \mathrm{m}^{2}$. The piezoelectric properties were set to zero. The permittivity was set to $\kappa_{11}=\kappa_{22}=\kappa_{33}=$ $3.542 \cdot 10^{-11} \mathrm{~F} / \mathrm{m}$.

A thin piezoelectric plate of $1 \mathrm{~mm} \times 1 \mathrm{~mm} \times 50 \mu \mathrm{m}$ was also modeled to obtain the reference values of a piezoelectric 


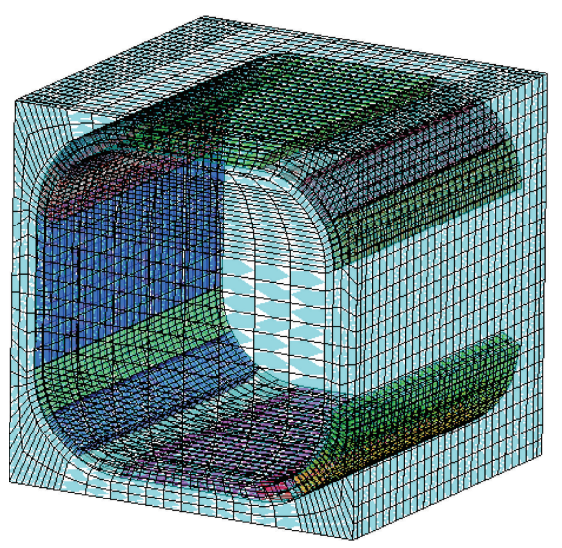

$1.1 \mathrm{~mm}$ by $1.1 \mathrm{~mm}$ by $1.1 \mathrm{~mm}$ PZT-5A sensor $50 \mu \mathrm{m}$ thick

(a)

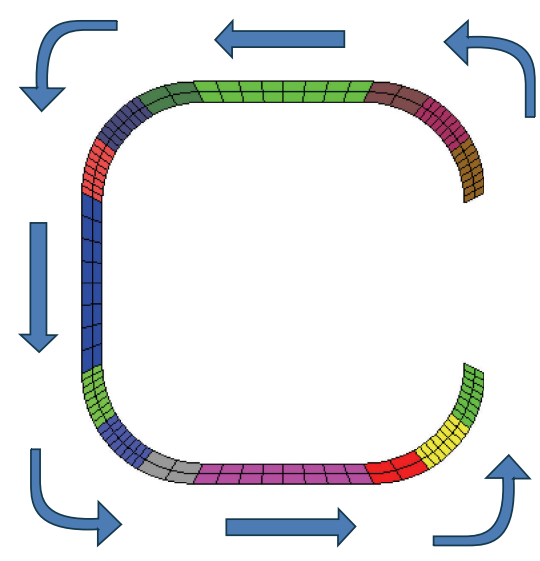

PZT-5A sensor with polarization orientations shown by arrows

(b)

Figure 1: C-shaped embedded sensor design. The parts of the C-shaped sensor with different colours have different orientations of polarization which follows the contour of the C-shaped element.

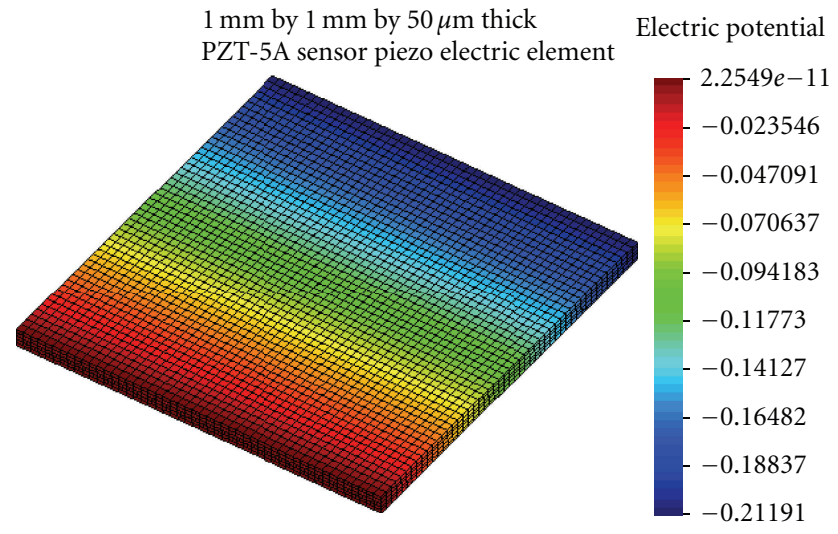

Figure 2: Piezoelectric plate under a hydrostatic pressure of $100,000 \mathrm{~Pa}$. Electric potential generated due to the application of a hydrostatic pressure field.

response as shown in Figure 2. The boundary conditions on the plate were applied to prevent a rigid body motion and rotation. The piezoelectric material was defined using PZT$5 \mathrm{~A}$ elements and polarized along one side of the thin square plate. A zero volt electric potential was assigned at a single node at the corner of the piezoelectric plate.

\section{Results}

The C-shaped piezoelectric sensor polarized along the perimeter and embedded in an epoxy matrix under a hydrostatic pressure field was analyzed using FEA. The results of this study demonstrated the formation of an internal bending mode in the piezoelectric $\mathrm{C}$-shaped sensor element. The bending mode is produced in all locations of the C-shaped sensor element as shown in Figure 3(a). The electric potential difference generated in this sensor design was approximately $11.5 \mathrm{~V}$ as shown in Figure 3(b). This high potential difference is approximately $11.3 \mathrm{~V}$ greater than that of a thin piezoelectric plate of the same thickness polarized along its length under the same hydrostatic pressure (Figure 2). This represents an increase in electric potential difference by a factor of approximately fifty seven times when compared to that of a thin piezoelectric plate. The electric potential distribution on the C-shaped sensor element shown in Figure 3(b) indicates that the electrodes should be placed at the tips of the C-shaped element. However, the ability to polarize a C-shaped sensor element along its length would present a challenge during manufacturing and would require that multiple interdigitated electrodes be placed at every corner of the sensor. Another challenge presented by this type of sensor is its manufacturing. The C-Shape piezoelectric component could be micromachined using lasers as it has been shown in [22]. Other authors have shown the use of etching processes for micromachining of bulk piezoelectric substrates [23]. Although, these etching processes are mostly applied to thin films, it is foreseeable that thin C-shaped film sensors could be manufactured for later encapsulation into an epoxy matrix. Finally, a Ceramic Injection Molding combined with sintering and micromachining could be used to produce the basic shape of the sensor as described in [24]. The electrodes could then be printed on the sensor through deposition technology as it is commonly performed in the semiconductor and electronic industry. The final step of the manufacturing process would be the attachment of miniaturized wires to the sensor electrodes for polarization and signal reception. The sensor itself would then be surrounded by an epoxy matrix to protect the sensor and provide it with resilience of a composite structure as well as the required interaction between the piezoelectric element and the epoxy matrix necessary for the augmentation of the electric potential output described in this study. 


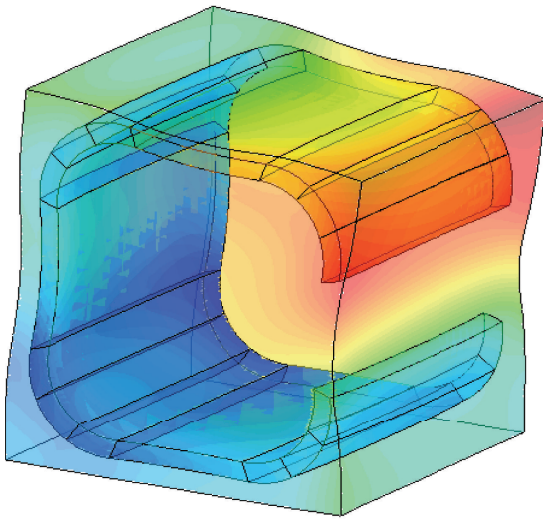

$1.1 \mathrm{~mm}$ by $1.1 \mathrm{~mm}$ by $1.1 \mathrm{~mm}$

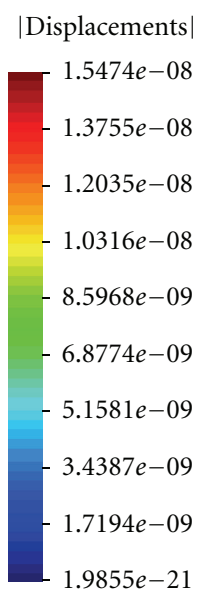

(a)

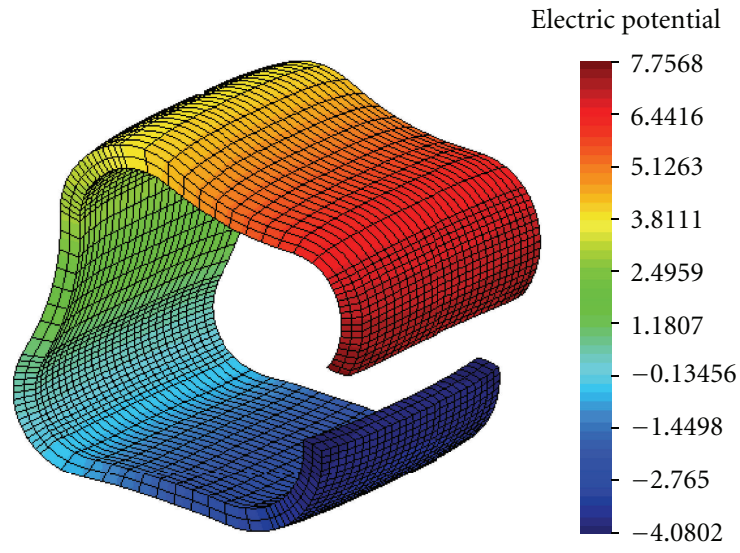

$50 \mu \mathrm{m}$ thick PZT-5A sensor polarized along the perimeter

(b)

Figure 3: (a) Piezoelectric C-ring sensor polarized along the contour under the hydrostatic pressure of 100,000 Pa. (b) Piezoelectric Cring sensor with the epoxy matrix removed from the figure in order to visualize the electric potential distribution on the surface of the piezoelectric material.

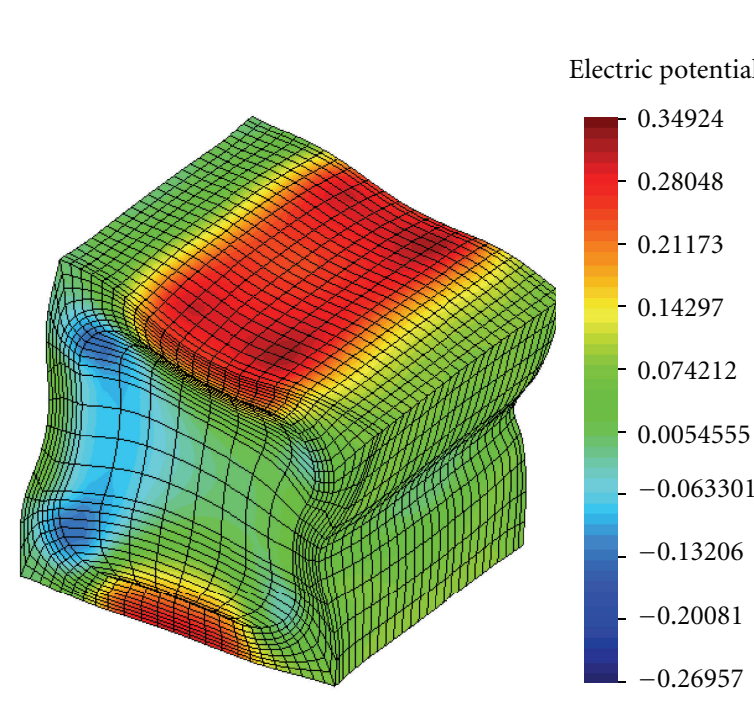

(a)

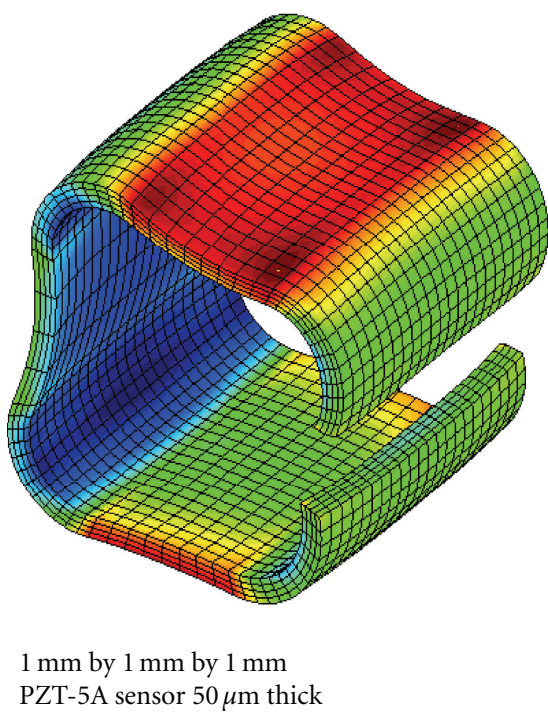

(b)

Figure 4: (a) Piezoelectric C-ring sensor polarized through the thickness under the hydrostatic pressure load of 100,000 Pa in the epoxy matrix. (b) Piezoelectric C-ring sensor with the epoxy matrix removed from the figure in order to visualize the electric potential distribution on the surface of the piezoelectric material.

The second set of simulations analyzed the performance of the same C-shaped piezoelectric sensor element with a through-thickness polarization. A through thickness polarization would only require two electrodes placed on the inner and outer surfaces of the $\mathrm{C}$-shaped sensor element and would make the sensor easier to manufacture since miniaturized interdigitized electrodes through the perimeter of the sensor would not be required. The results shown in Figures 4(a)4(b) indicate that the deformed sensor produces an electric potential of approximately $0.6 \mathrm{~V}$. This electric potential is significantly lower than it would be if the sensor was polarized along its circumference. However, it has also been shown that the electric potential output is still approximately three times greater than that of a single thin piezoelectric plate under the same hydrostatic pressure.

\section{Conclusions}

The results of this study indicate that an enhanced electric potential output from a composite sensor with a C-shaped piezoelectric element can be obtained. This larger signal output is achieved through a combination of bending 
deformation of the piezoelectric element and polarization orientation along the piezoelectric material portion of the sensor. This study demonstrates that a through thickness polarization orientation, while presenting benefits from a manufacturing point of view, results in a much lower signal output level. It has been shown that pressure sensors making use of piezoelectric materials can substantially increase their electric potential output by optimizing their deformation mode and material polarization distribution. Although this study has not optimized the shape and size of the sensor, it has been demonstrated that an electric potential signal could be increased by a factor of 3 to 57 , based on the sensor shape design and the polarization of the material in comparison to a single flat plate made of the same piezoelectric material.

\section{References}

[1] R. A. Clark and E. Reissner, "Deformations and stresses in bourdon tubes," Journal of Applied Physics, vol. 21, no. 12, pp. 1340-1341, 1950.

[2] P. J. Chen, D. C. Rodger, R. Agrawal et al., "Implantable micromechanical parylene-based pressure sensors for unpowered intraocular pressure sensing," Journal of Micromechanics and Microengineering, vol. 17, no. 10, pp. 1931-1938, 2007.

[3] W. N. MacPherson, E. J. Rigg, J. D. C. Jones, V. V. R. K. Kumar, J. C. Knight, and P. St. J. Russell, "Finite-element analysis and experimental results for a microstructured fiber with enhanced hydrostatic pressure sensitivity," Journal of Lightwave Technology, vol. 23, no. 3, pp. 1227-1231, 2005.

[4] W. Parkes, V. Djakov, J. S. Barton et al., "Design and fabrication of dielectric diaphragm pressure sensors for applications to shock wave measurement in air," Journal of Micromechanics and Microengineering, vol. 17, no. 7, pp. 1334-1342, 2007.

[5] X. D. Wang and G. L. Huang, "The coupled dynamic behavior of piezoelectric sensors bonded to elastic media," Journal of Intelligent Material Systems and Structures, vol. 17, no. 10, pp. 883-894, 2006.

[6] V. Shamanna, S. Das, Z. Çelik-Butler, D. P. Butler, and K. L. Lawrence, "Micromachined integrated pressure-thermal sensors on flexible substrates," Journal of Micromechanics and Microengineering, vol. 16, no. 10, pp. 1984-1992, 2006.

[7] S. Son and N. C. Goulbourne, "Finite deformation of tubular dielectric elastomer sensors," Journal of Intelligent Material Systems and Structures, vol. 20, pp. 2187-2199, 2009.

[8] C. Pramanik, H. Saha, and U. Gangopadhyay, "Design optimization of a high performance silicon MEMS piezoresistive pressure sensor for biomedical applications," Journal of Micromechanics and Microengineering, vol. 16, no. 10, pp. 2060-2066, 2006.

[9] G. Gautschi, Piezoelectric Sensorics: Force, Strain Pressure, Acceleration and Acoustic Emission Sensors, Materials and Amplifiers, Springer, Berlin, Germany, 2002.

[10] K. Fricke and H. L. Hartnagel, "Pressure measurement by GaAs piezoelectric sensors," Electronics Letters, vol. 26, no. 11, pp. 693-694, 1990.

[11] L. C. Chin, V. V. Varadan, and V. K. Varadan, "Finite element analysis of flextensional electroacoustic transducers," in Ultrasonic Symposium, pp. 481-484, 1991.

[12] P. Mane, K. Mossi, and R. Bryant, "Experimental design and analysis for piezoelectric circular actuators in flow control applications," Smart Materials and Structures, vol. 17, no. 1, Article ID 015013, 2008.
[13] P. Schiller, D. L. Polla, and M. Ghezzo, "Surfacemicromachined piezoelectric pressure sensors," in Proceedings of the IEEE Solid-State Sensor and Actuator Workshop, pp. 187-190, June 1990.

[14] M. Akiyama, Y. Morofuji, T. Kamohara et al., "Flexible piezoelectric pressure sensors using oriented aluminum nitride thin films prepared on polyethylene terephthalate films," Journal of Applied Physics, vol. 100, no. 11, Article ID 114318, 2006.

[15] J. Song, J. Zhou, and Z. L. Wang, "Piezoelectric and semiconducting coupled power generating process of a single $\mathrm{ZnO}$ belt/wire. A technology for harvesting electricity from the environment," Nano Letters, vol. 6, no. 8, pp. 1656-1662, 2006.

[16] Y. Gao and Z. L. Wang, "Electrostatic potential in a bent piezoelectric nanowire. The fundamental theory of nanogenerator and nanopiezotronics," Nano Letters, vol. 7, no. 8, pp. 24992505, 2007.

[17] Z. L. Wang and J. Song, "Piezoelectric nanogenerators based on zinc oxide nanowire arrays," Science, vol. 312, no. 5771, pp. 242-246, 2006.

[18] B. J. Cannon and D. Brei, "Feasibility study of microfabrication by coextrusion (MFCX) hollow fibers for active composites," Journal of Intelligent Material Systems and Structures, vol. 11, no. 9, pp. 659-670, 2001.

[19] J. F. Fernandez, A. Dogan, Q. M. Zhang, J. F. Tressler, and R. E. Newnham, "Hollow piezoelectric composites," Sensors and Actuators A, vol. 51, no. 2-3, pp. 183-192, 1996.

[20] M. Martinez and A. Artemev, "Finite element analysis of broken fiber effects on the performance of active fiber composites," Composite Structures, vol. 88, no. 3, pp. 491-496, 2009.

[21] W. P. Eaton and J. H. Smith, "Micromachined pressure sensors: review and recent developments," Smart Materials and Structures, vol. 6, no. 5, pp. 530-539, 1997.

[22] D. Most, J. Choi, L. J. Belenky, and C. B. Eom, "Laser micromachining of $\mathrm{SrTiO}_{3}$ single crystal," Solid-State Electronics, vol. 47, no. 12, pp. 2249-2253, 2003.

[23] T. Srinivas, "Piezoelectric microelectromechanical systemschallenges and opportunities," Procedia Engineering, vol. 5, pp. 468-471, 2010.

[24] I. Stanimirović and Z. Stanimirović, "Piezoelectric ceramics by powder injection molding," in Procedings of the 27th International Conference on Microelectronics (MIEL '10), pp. 231-233, Niš, Serbia, May 2010. 

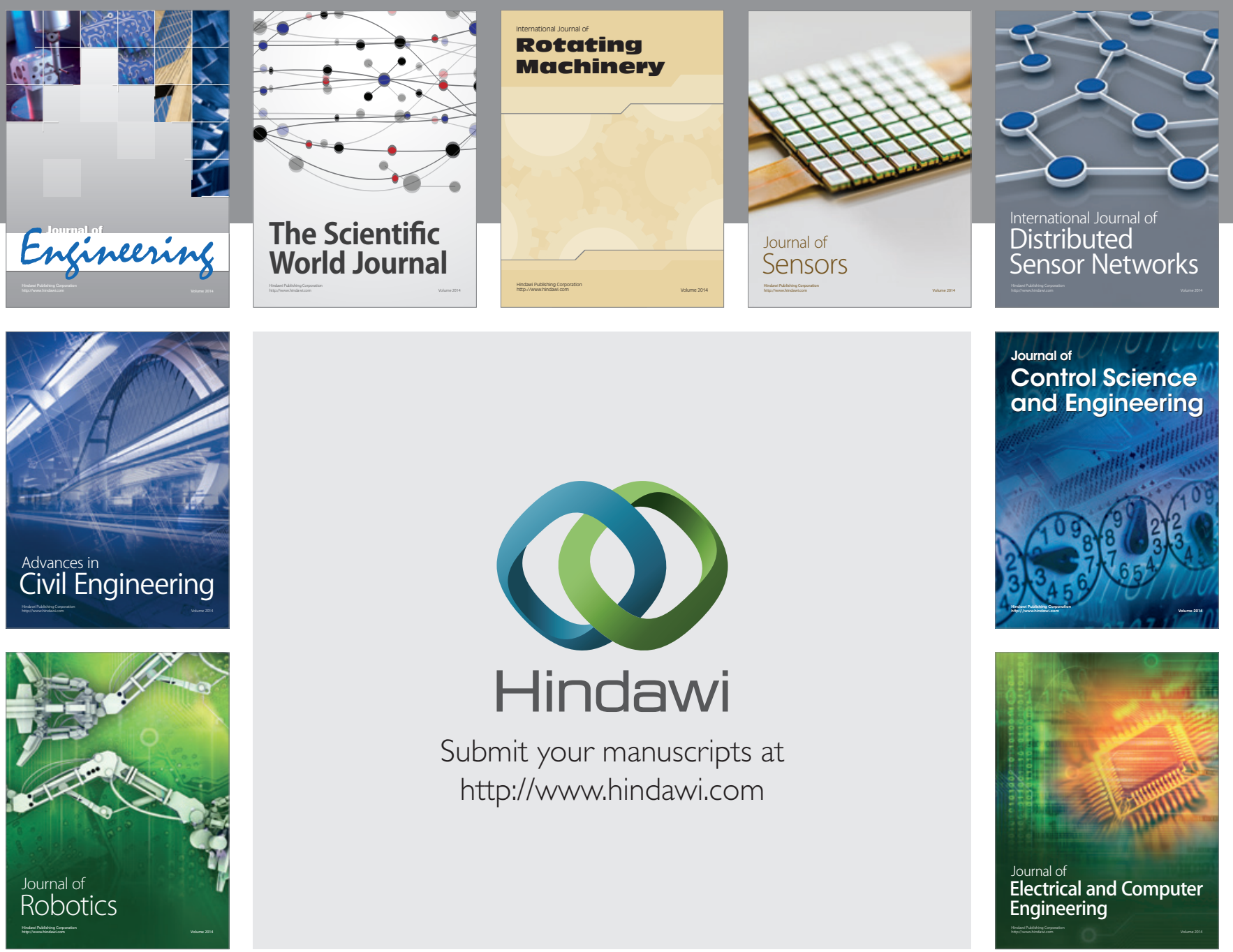

Submit your manuscripts at

http://www.hindawi.com
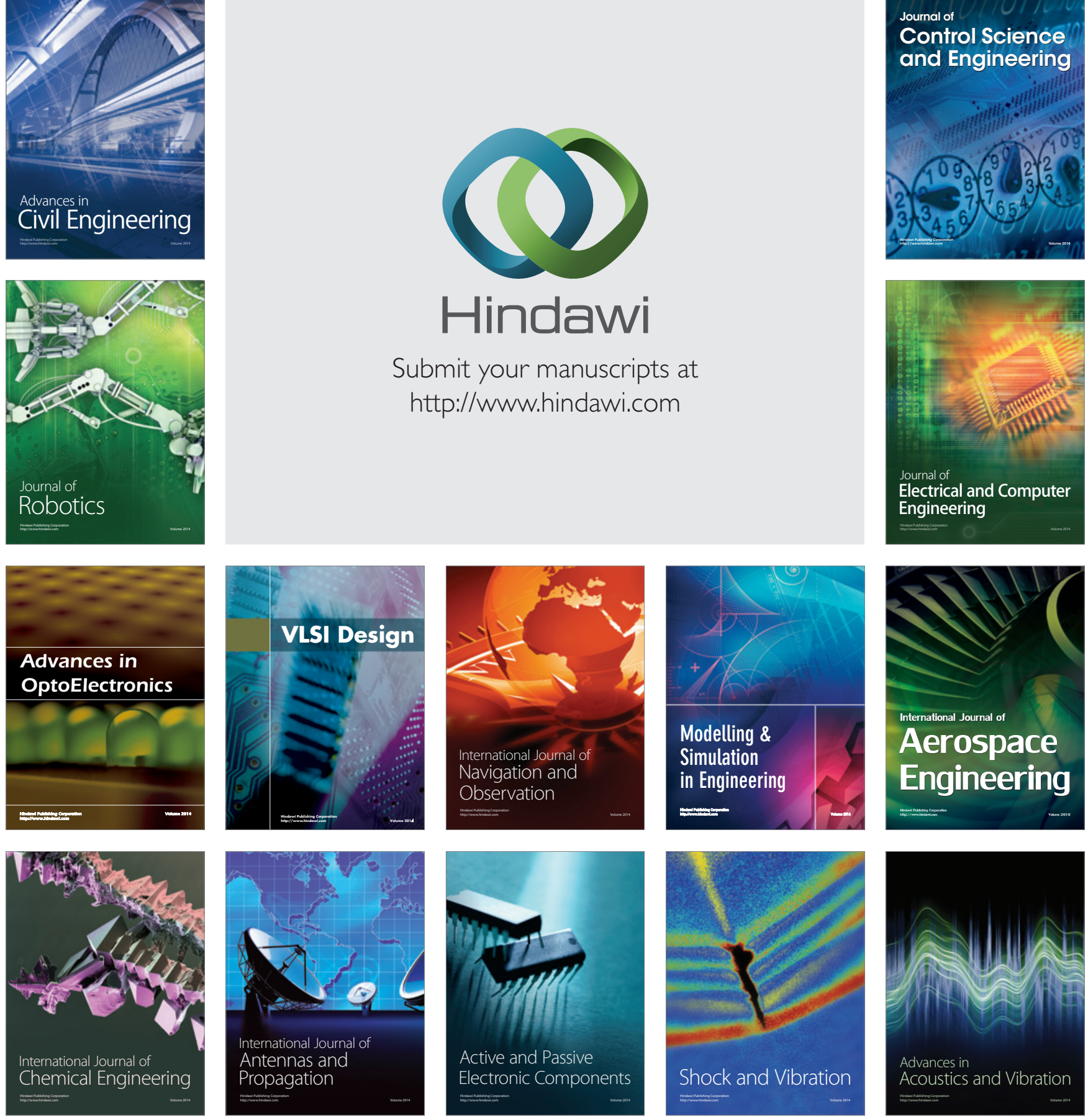